\title{
BOUNDEDNESS OF THE MAXIMAL OPERATOR ON WEIGHTED BMO
}

\author{
STEVEN BLOOM
}

\begin{abstract}
The Hardy-Littlewood maximal operator $M^{*}$ is a bounded operator mapping $\mathrm{BMO}_{w}$ into $\mathrm{BLO}_{w}$ if and only if the weight $w$ is a Reverse Hölder weight in weak $\alpha_{2}$.
\end{abstract}

$\mathbf{T}$ will denote the unit circle in the complex plane. For an interval $I \subset \mathbf{T}, I(f)=$ $(1 /|I|) \int_{I} f(x) d x$. A weight $w \in \mathrm{RH}$ if $w$ satisfies the Reverse Hölder Inequality: There exists a $p>1$ and a constant $C$ such that $I\left(w^{p}\right)^{1 / p} \leq C I(w)$ for all intervals I. $f$ is a function of weighted bounded mean oscillation, $f \in \mathrm{BMO}_{w}$, provided

$$
I(|f-I(f)|) \leq C T(w) \text { for all intervals } I,
$$

while $f$ is a function of weighted bounded lower oscillation, $f \in \mathrm{BLO}_{w}$, if

$$
I(f)-\underset{I}{\operatorname{essinf} f \leq C I(w)} \text { for all } I .
$$

A weight $w$ belongs to the Kerman-Torchinsky class $\alpha_{p}$ if for any interval $I$ and measurable set $E \subset I[\mathbf{5}]$,

$$
\left(\frac{|E|}{|I|}\right)^{p} \leq C \frac{w(E)}{w(I)} .
$$

$\alpha_{p} \subset \mathrm{RH}$. Indeed, $A_{p} \subset \alpha_{p} \subset \bigcap_{q>p} A_{q}$, where $A_{p}$ is the Muckenhoupt class of weights. In [2], we introduced the class weak $\alpha_{p}$, defined by (1) with the additional proviso that $E$ be an interval. This class properly contains $\alpha_{p}$. For if $w$ is a doubling measure with constant $2^{p}$, that is

$$
w(J) \leq 2^{p} w(I) \text { for all intervals } I \subset J
$$

with $|J|=2|I|$, then a standard argument shows $w \in$ weak $\alpha_{p}$. But there exist doubling measures that are not in $\mathrm{RH}[4]$. It is not yet known whether $\alpha_{p}$ and $\mathrm{RH} \cap$ weak $\alpha_{p}$ are the same.

Let $M^{*}$ denote the Hardy-Littlewood maximal operator

$$
M^{*} f(x)=\sup _{I \ni x} I(|f|) .
$$

We will show the following result.

THEOREM. $M^{*}: \mathrm{BMO}_{w} \rightarrow \mathrm{BLO}_{w}$ is a bounded operator if and only if $w \in$ $\mathrm{RH} \cap$ weak $\alpha_{2}$.

The necessity of the condition $\mathrm{RH} \cap$ weak $\alpha_{2}$ was proven in [2], but a stronger condition was used for the converse. We prove the sufficiency here:

Received by the editors June 19, 1984 .

1980 Mathematics Subject Classification. Primary 42B25; Secondary 42A50.

(C) 1985 American Mathematical Society $0002-9939 / 85 \$ 1.00+\$ .25$ per page 
Fix an interval $I$, and assume, with no loss of generality, that $f \geq 0, f \in \mathrm{BMO}_{w}$. For $x \in I$, define

$$
F_{1}(x)=\sup \{J(f): x \in J, J \subset 4 I\}, \quad F_{2}(x)=\sup \{J(f): x \in J, J \not \subset 4 I\},
$$

where $4 I$ is the interval concentric with $I$ but of four times the length. So on $I, M^{*} f(x)=\max \left\{F_{1}(x), F_{2}(x)\right\}$. Put

$$
E_{1}=\left\{x \in I: F_{1}(x) \geq F_{2}(x)\right\}, \quad E_{2}=\left\{x \in I: F_{2}(x)>F_{1}(x)\right\}
$$

and $\beta=\operatorname{essinf}_{I} M^{*} f$.

It will suffice to show

$$
\frac{1}{|I|} \int_{E_{i}}\left(F_{i}-\beta\right) \leq C I(w) \text { for } i=1 \text { and } 2 .
$$

For $i=1$, following the proof in [2], we obtain disjoint intervals $I_{n} \subset 4 I$, intervals $J_{n} \supset I_{n}$ of twice their length, and a "bad" function

$$
b=\sum_{n}\left(f-J_{n}(f)\right) \chi_{I_{n}} .
$$

The proof of (2) requires showing $b \in L^{r}$ for some $r>1$ with

$$
\|b\|_{r} \leq C\left(\int_{4 I} w^{r}\right)^{1 / r}
$$

As $w \in \mathrm{RH}$, there exists an $r>1$ and $p>1$ such that $w^{r} \in A_{p}, w^{-r q / p} \in A_{q}$, where $1 / p+1 / q=1$, and $J\left(w^{r}\right) \leq C J(w)^{r}$ for all intervals $J[3]$. Now

$$
\begin{aligned}
\|b\|_{r}^{r} & =\sum_{n} \int_{I_{n}}\left|f-J_{n}(f)\right|^{r} w^{-r / p} w^{r / p} \\
& \leq \sum_{n}\left(\int_{J_{n}}\left|f-J_{n}(f)\right|^{q r} w^{-q r / p}\right)^{1 / q}\left(\int_{J_{n}} w^{r}\right)^{1 / p} .
\end{aligned}
$$

Let $f_{n}^{\sharp}(x)=\sup \left\{J|f-J(f)|: x \in J \subset J_{n}\right\}$ and $w_{n}^{*}(x)=\sup \left\{J(w): x \in J \subset J_{n}\right\}$; that is, the sharp and maximal functions restricted to $J_{n}$. By the weighted version of the Sharp Function Theorem [1],

$$
\left(\int_{J_{n}}\left|f-J_{n}(f)\right|^{q r} w^{-q r / p}\right)^{1 / q} \leq C\left(\int_{J_{n}}\left(f_{n}^{\sharp}\right)^{q r} w^{-q r / p}\right)^{1 / q} .
$$

But with $f \in \mathrm{BMO}_{w}, f_{n}^{\sharp}(x) \leq C w_{n}^{*}(x) \leq C\left[\left(w^{r}\right)_{n}^{*}(x)\right]^{1 / r}$, using Hölder's Inequality. This, Muckenhoupt's Theorem, and the fact that $w^{r}$ is a doubling measure give

$$
\begin{aligned}
\|b\|_{r}^{r} & \leq C \sum_{n}\left(\int_{J_{n}}\left[\left(w^{r}\right)_{n}^{*}\right]^{q} w^{-q r / p}\right)^{1 / q}\left(\int_{J_{n}} w^{r}\right)^{1 / p} \\
& \leq C \sum_{n} \int_{J_{n}} w^{r} \leq C \sum_{n} \int_{I_{n}} w^{r} \leq C \int_{4 I} w^{r}
\end{aligned}
$$

which is (3).

For $i=2$, we actually show

$$
F_{2}(x)-\beta \leq C I(w) \text { for all } x \in E_{2}
$$


which gives (2). We analyze the case where $x \in E_{2} \cap J, J \not \subset 4 I, I=[a, a+h]$ and $J=[b, c]$ with $b \in I$. Set $\bar{J}=[b-h, c], J_{0}=[b-h, b]$ and $J_{k}=\left[b, b+2^{k-1} h\right]$ as long as $2^{k-1} h<c-b$. Let $n$ be the smallest integer with $2^{n-1} h \geq c-b$, and call $J=J_{n}$. Since $I \subset \bar{J}, \bar{J}(f) \leq \beta$. Hence,

$$
\begin{aligned}
J(f)-\beta & \leq J(f)-\bar{J}(f)=\left(\frac{1}{|J|}-\frac{1}{|\bar{J}|}\right) \int_{J} f-\frac{1}{|\bar{J}|} \int_{J_{0}} f \\
& =\frac{h}{|\bar{J}|}\left[J(f)-J_{0}(f)\right] \\
& \leq 2^{2-n}\left[J_{1}(f)-J_{0}(f)+J_{n}(f)-J_{1}(f)\right] .
\end{aligned}
$$

Now let $R=J_{0} \cup J_{1}$. Then

$$
\begin{aligned}
\left|J_{1}(f)-J_{0}(f)\right| & \leq\left|J_{1}(f)-R(f)\right|+\left|J_{0}(f)-R(f)\right| \leq \frac{4}{|R|} \int_{R}|f-R(f)| \\
& \leq C R(w) \quad \text { since } f \in \mathrm{BMO}_{w} .
\end{aligned}
$$

Also,

$$
\begin{aligned}
\left|J_{n}(f)-J_{1}(f)\right| & =\left|\sum_{k=1}^{n-1} J_{k+1}(f)-J_{k}(f)\right| \\
& \leq 2 \sum_{k=1}^{n-1} J_{k+1}\left(\left|f-J_{k+1}(f)\right|\right) \leq C \sum_{k=1}^{n-1} J_{k+1}(w) .
\end{aligned}
$$

By the weak $\alpha_{2}$ condition,

$$
J_{k+1}(w) \leq C \frac{\left|J_{k+1}\right|}{\left|J_{1}\right|} J_{1}(w) \leq C 2^{k} J_{1}(w) .
$$

Hence,

$$
\left|J_{n}(f)-J_{1}(f)\right| \leq C J_{1}(w) \sum_{k=1}^{n-1} 2^{k} \leq C 2^{n+1} R(w) .
$$

Thus

$$
J(f)-\beta \leq 2^{2-n}\left[C_{1} R(w)+C_{2} 2^{n+1} R(w)\right] \leq C R(w) .
$$

But $w$ is a doubling measure [3], so $R(w) \leq C I(w)$ and (4) follows.

\section{REFERENCES}

1. S. Bloom, A commutator theorem and weighted BMO (to appear).

2. - The maximal function on weighted BMO, Harmonic Analysis (Proc. Conf., Cortona, Italy, 1982), pp. 227-239.

3. R. R. Coifman and C. Fefferman, Weighted norm inequalities for maximal functions and singular integrals, Studia Math. 51 (1974), 241-250.

4. C. Fefferman and B. Muckenhoupt, Two nonequivalent conditions for weight functions, Proc. Amer. Math. Soc. 45 (1974), 99-104.

5. R. A. Kerman and A. Torchinsky, Integral inequalities with weights for the Hardy maximal function, Studia Math. 71 (1981/82), 277-284.

Department of Mathematics, Siena College, loudonville, New York 12211 